Research Paper

\title{
Verbal Fluency Performance in Normal Adult Population in Iran: Norms and Effects of Age, Education, and Gender
}

\author{
Saeedeh Shirdel ${ }^{1}$ (D), Setareh Esmaeeli ${ }^{1}$, Kaveh Alavi² (D), Pezhman Ghaemmaghami ${ }^{3}$, Seyed Vahid Shariat ${ }^{2 *}$ (iD \\ 1. School of Medicine, Iran University of Medical Sciences, Tehran, Iran. \\ 2. Mental Health Research Center, School of Behavioral Sciences and Mental Health (Tehran Institute of Psychiatry), Iran University of Medical Sci- \\ ences, Tehran, Iran. \\ 3. Iran Psychiatric Hospital, Iran University of Medical Sciences, Tehran, Iran.
}

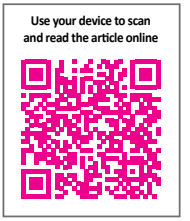

Citation Shirdel, S., Esmaeeli, S., Alavi, K., Ghaemmaghami, P., Shariat, S. V. Verbal Fluency Performance in Normal Adult Population in Iran: Norms and Effects of Age, Education, and Gender. Basic and Clinical Neuroscience, 13(1), 129-138. http:// dx.doi.org/10.32598/bcn.2021.363.1

http://dx.doi.org/10.32598/bcn.2021.363.1

\section{(i) (3)}

Article info:

Received: 17 Jan 2019

First Revision: 05 Mar 2020

Accepted: 24 Aug 2020

Available Online: 01 Jan 2022
Keywords:

Neuropsychological tests, Verbal fluency, Persian, Category, Phonemic

\begin{abstract}
$\underline{\text { ABS T RACT }}$
Introduction: Verbal fluency is a cognitive function that can be easily assessed at the bedside and provide valuable data for clinical assessment of various cognitive functions. We decided to provide a standardized test to assess verbal fluency in the Persian language, including both phonemic and semantic fluency subtests.

Methods: First, three phonemes (/p/, /d/, and / $/$ ) and three categories (animals, fruits, and kitchen appliances) were selected based on a pilot study and a panel of specialists. Then, we selected 500 Persian-speaking participants ( $47.8 \%$ male) aged 18 to 65 years via a convenient sampling method from the general population. Participants were grouped according to age, gender, and education. They performed the verbal fluency test.

Results: The Mean \pm SD number of generated words in letter fluency and semantic fluency was $8.3 \pm 4.1$ and $18.0 \pm 5.5$, respectively. Age, educational level, and mother tongue were associated with letter fluency. Semantic fluency was associated with age, gender, education level, and mother tongue.

Conclusion: For a more reliable clinical assessment, we suggest using all three letters (phonemes) and three semantic categories for each subject, calculating the mean of the produced words, and comparing them with the suggested cut-off points provided for each subcategory. Age was negatively correlated with the number of generated words in letter fluency $(\mathrm{r}=-0.33 ; \mathrm{P}<0.001)$ and semantic fluency tasks $(r=-0.26 ; \mathrm{P}<0.001)$. In the letter fluency task, there was no statistically significant difference between males and females according to the number of generated words $(\mathrm{P}=0.057)$. However, in semantic fluency, female participants generated more words $(\mathrm{P}=0.005)$. Mother tongue (Farsi) showed a significant effect both on letter fluency $(t=5.55, P<0.001)$ and semantic fluency $(\mathrm{t}=9.41, \mathrm{P}<0.001)$. Level of education had a significant association with both letter fluency $(\mathrm{F}=117.23, \mathrm{P}<0.001)$ and semantic fluency $(\mathrm{F}=64.48, \mathrm{P}<0.001)$.
\end{abstract}

\section{* Corresponding Author:} Seyed Vahid Shariat, Professor.

Address: 2. Mental Health Research Center, School of Behavioral Sciences and Mental Health (Tehran Institute of Psychiatry), Iran University of Medical Sciences, Tehran, Iran.

Tel: +98 (21) 66506862

E-mail: shariat.v@iums.ac.ir ; vahid.shariat@gmail.com 


\section{Highlights}

- The study subjects generated $8.3 \pm 4.1$ words in one minute in the letter fluency test.

- Letter fluency was associated with educational level and mother tongue.

- The Mean \pm SD number of generated words in semantic fluency (18 \pm 5.5$)$ was higher than letter fluency.

Semantic fluency was associated with age, gender, education level, and mother tongue.

\section{Plain Language Summary}

Practitioners use neuropsychological tests to diagnose mental problems. Verbal fluency is a test in which participants have to generate as many words as possible from a specified category in a given time. This category can be phonemic (letter), which means words beginning with a specified letter or semantic, including objects such as animals or fruits. The number of words produced by participants is essential, and if it is fewer than normal, it shows psychological or neurological conditions such as Alzheimer disease. The norms are variable in different languages, cultures, and educational levels. We found that the Mean \pm SD numbers of generated words in letter fluency and semantic fluency were $8.3 \pm 4.1$ and $18.0 \pm 5.5$, respectively. These values can be used for neuropsychological testing in the Iranian population.

\section{Introduction}

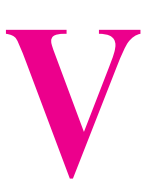

erbal fluency is a cognitive function mainly based on information retrieval from memory. Successful retrieval requires executive control over cognitive processes such as selective attention, response inhibition, mental set shifting, internal response generation, and self-monitoring (Patterson,

2011). The verbal fluency test is widely used for cognitive assessment during neuropsychological assessment. The test includes phonemic fluency and semantic fluency. In phonemic fluency, participants should generate as many words as possible with a specified initial letter (phoneme) in a given time (60 s) (Patterson, 2011). In semantic fluency, participants should name as many words as they can from a specific category, such as animals or fruits, in a given time (60 s) (Patterson, 2011). The number of generated words and objects is recorded and compared with normative data. The normative data is different among different languages and cultures; therefore, the test has been changed to some extent in different countries according to the local characteristics and language necessities. (Olabarrieta-Landa, et al., 2015; Ostrosky-Solis, Gutierrez, Flores, \& Ardila, 2007).

A previous study has provided preliminary normative data for a Persian verbal fluency test (Hasemian-Shirvan, Shirazi, Aminikhoo, Zareaan, \& Ekhtiari, 2018). However, the authors have selected letters solely based on their frequency and overlooked other important deter- mining factors. Furthermore, their sample size w limited to 50 and 100 subjects.

We performed this study in Iran to localize the verbal fluency test in the Persian language and determine a suitable set of letters for phonemic fluency and semantic categories for the semantic fluency part. We provided normative data based on education and age subgroups and provided cut-off points for clinical use.

\section{Methods}

\section{Letter and category selection}

For letter (phoneme) selection, first, we omitted the following letters: those with a low frequency as word initials (ein ,f,j, ch,jh); those that were initials to frequent socially inappropriate words $(\mathrm{k}, \mathrm{g}, \mathrm{kh})$; those with the same sound (in Persian several letters have the same sound) (s, t, z, gh, h); and those that are a beginning letter for some common verb forms including present continuous, conditionals, and negative forms $(n, b)$. Then, we performed a pilot study with the selected letters on 10 subjects with different educational levels to see which letters were appropriate. As a result, five phonemes $(/ \mathrm{p} /, / \mathrm{d} /, / \mathrm{g} /, / \mathrm{m} /, \mathrm{r} /)$ met our criteria. Finally, a panel of psychiatrists and researchers selected three letters assumed to be more appropriate for our purpose (Persian phonemes: /p/, / / , and /d/). 
For the category selection, we first suggested ten semantic fields and performed a pilot study and then a group discussion to select the final three categories (including animals, fruits, and kitchen appliances).

\section{Determining the normal range}

\section{Study participants}

We used a convenient sampling method to recruit 500 subjects with the following inclusion criteria: aged 18 to 65 years, ability to talk in Farsi fluently, and no history of psychiatric or neurological illnesses (e.g. Parkinson disease, stroke) based on the subject's report. We also grouped the samples according to three variables that could probably affect the outcome, including education (elementary, high school, university), gender (female, male), and age (below and higher than 45 years). We asked the participants about their proficiency in other languages to determine bilingual participants. We intended to recruit 40 subjects in each group, reach a total sample size of 480 subjects, and rounded it up to 500 .

\section{Study procedure}

Tests were taken while the subjects sat at a desk in a relatively quiet environment. First, the test was described for each subject, and the instruction was given. Examples were provided for a better understanding of the instructions. The participants were told that they had one minute to say as many words with the specified initial or from the specified category. It was also mentioned that they should not say proper names, such as names of people and cities unless the subject intended the meaning of the name. Each participant was asked to generate six words; three for phonemic (/p/, /d/, and $/ \mathrm{J} /)$ and three for semantic fluency (animals, fruits, and kitchen appliances) parts. The order of word generation trials was not the same for all subjects. We supposed that the order of letters presented in the phonemic fluency test might affect the result in two possible ways. First, the participant might gradually warm up and perform better in the later letters. Second, cognitive fatigue might happen, and so the subject might have poorer performance in the later presented letters. To prevent these problems, we determined the six possible orders of the three letters and grouped the participants to perform the phonemic fluency test with the predefined order of one of the six sets of letters. Therefore, a roughly equivalent number of subjects performed the phonemic fluency test with each of the six sets of letters.
A trained medical student and a resident of psychiatry performed the tests and wrote down all of the generated words for each trial. To prevent any mistakes in the recording process, evaluators recorded the voice of subjects after asking for permission. If participants repeated a word, the person was not informed, but the word was counted only once. If a proper name was said the first time, the participant was reminded that proper names are not counted, but if the participant said that $\mathrm{s} /$ he meant the specific meaning of the name, it was counted.

\section{Data analysis}

Demographic data, including age, gender, educational level, and familiarity with other languages, were collected in a questionnaire developed by the research team. The obtained data were analyzed for possible associations between test scores and study variables using the $\mathrm{t}$ test and the Pearson correlation in SPSS (BM SPSS Statistics for Windows, Version 22.0. Armonk, NY: IBM Corp.). The normal range for phonemic and semantic fluency tests was also calculated for each gender and educational level. We calculate the lower 5th and 10th percentile for each subgroup based on gender (male or female), mother tongue (Farsi and other languages), and education levels (illiterate, primary or high school, and university education). As we had no standardized tool to compare the results of the current fluency test with other studies, we used the lower 5th and 10th percentile to suggest a cut-off point to differentiate the sound result of the test and possibly disordered ones. . It is noteworthy that this method has been used by some other researchers (Zarino, Crespi, Launi, Casarotti, 2014).

\section{Results}

A total of 500 subjects with a Mean \pm SD age of $40.9 \pm 12.7$ years participated in this study (Table 1). Twenty-one subjects $(4.2 \%)$ had a history of psychotic disorders, and 10 subjects $(2.0 \%)$ had a history of neurotic disorders. The Mean \pm SD numbers of generated words in letter fluency and semantic fluency were $8.3 \pm 4.1$ and $18.0 \pm 5.5$, respectively (Table 2 ). There were strong correlations between the numbers of words generated with the three different letters in the letter fluency task (0.7600.724-0.727). However, in the three different categories of semantic fluency tasks, numbers of generated words were moderately correlated $(0.651,0.642,0.705)$. The correlation coefficient between generated words in letter fluency and semantic fluency was 0.71 (Table 3 ).

Age was negatively correlated with the number of generated words in letter fluency $(\mathrm{r}=-0.33 ; \mathrm{P}<0.001)$ and 
Table 1. Demographic characteristics of the study participants

\begin{tabular}{ccc}
\hline & & No.(\%) \\
\hline Gender & Males & $239(47.8)$ \\
& Illiterate & $128(25.6)$ \\
Education & Preliminary & $127(25.4)$ \\
& High school & $118(23.6)$ \\
& University & $127(25.4)$ \\
\hline Mother tongue & Farsi & $344(68.4)$ \\
& Azari & $97(19.4)$ \\
& Gilaki & $20(4.0)$ \\
& Lori & $17(3.4)$ \\
\hline
\end{tabular}

"Including Taati, Afghani, Mashhadi, and Armani.

NEUR SCIENCE

semantic fluency tasks $(\mathrm{r}=-0.26 ; \mathrm{P}<0.001)$. In the letter fluency task, there was not a statistically significant difference between males and females in terms of the number of generated words. However, regarding semantic fluency, female participants generated more words $(\mathrm{t}=2.79, \mathrm{P}=0.005)$. In both tasks, individuals whose mother tongue was Farsi generated more words (letter fluency $[\mathrm{t}=5.55, \mathrm{P}<0.001]$ and semantic fluency $[\mathrm{t}=9.41$, $\mathrm{P}<0.001])$ within one minute. Also, individuals with university education generated more and illiterate individuals generated fewer words than the other groups' letter fluency $(\mathrm{F}=117.23, \mathrm{P}<0.001)$ and semantic fluency $(\mathrm{F}=64.48, \mathrm{P}<0.001)$. However, there was no statistically significant difference between individuals with primary education and those with high school levels of education. Therefore, we merged the two subgroups for further analyses (Table 4). Descriptions of the number of generated words within 12 groups are available upon request.

Table 2. Words generated in letter and semantic fluency tasks

\begin{tabular}{cccccccccc}
\hline & \multicolumn{4}{c}{ Letter Fluency } & \multicolumn{5}{c}{ Semantic Fluency } \\
\cline { 2 - 9 } Variables & $/ \mathbf{p} /$ & $/ \mathrm{d} /$ & $/ \mathrm{J} /$ & Mean & Animals & Fruits & Kitchen & Mean \\
\hline Mean \pm SD & $8.4 \pm 4.5$ & $8.5 \pm 4.6$ & $8.1 \pm 4.3$ & $8.3 \pm 4.1$ & $17.7 \pm 6.4$ & $18.3 \pm 6.3$ & $17.9 \pm 6.2$ & $18.0 \pm 5.5$ \\
\hline Minimum & 0 & 0 & 0 & 0 & 4 & 1 & 3 & 3.0 \\
\hline $5^{\text {th }}$ percentile & 2 & 1 & 2 & 2 & 8 & 7 & 8 & 8.3 \\
\hline $10^{\text {th }}$ percentile & 3 & 2 & 3 & 2.7 & 10 & 10 & 10 & 11.0 \\
\hline $25^{\text {th }}$ percentile & 5 & 5 & 5 & 5.3 & 13 & 14 & 14 & 14.3 \\
\hline $50^{\text {th }}$ percentile & 8 & 8 & 8 & 8.3 & 17 & 18 & 18 & 18.3 \\
\hline $75^{\text {th }}$ percentile & 11 & 12 & 11 & 11.0 & 22 & 22 & 22 & 21.7 \\
\hline $90^{\text {th }}$ percentile & 14 & 14 & 14 & 13.7 & 26 & 26 & 26 & 24.7 \\
\hline $95^{\text {th }}$ percentile & 17 & 17 & 15 & 15.0 & 29 & 28 & 28 & 27.3 \\
\hline Maximum & 23 & 22 & 23 & 20.7 & 41 & 49 & 40 & 34.7 \\
\hline
\end{tabular}


Table 3. Correlation between generated words with each letter and semantic category

\begin{tabular}{|c|c|c|c|c|c|c|}
\hline Variables & Letter: /p/ & Letter: /d/ & Letter: / // & $\begin{array}{c}\text { Semantic: } \\
\text { Animals }\end{array}$ & $\begin{array}{c}\text { Semantic: } \\
\text { Fruits }\end{array}$ & $\begin{array}{c}\text { Semantic: } \\
\text { Kitchen }\end{array}$ \\
\hline Letter: /p/ & 1 & & & & & \\
\hline Letter: /d/ & 0.760 & 1 & & & & \\
\hline Letter: /J/ & 0.724 & 0.727 & 1 & & & \\
\hline Semantic: Animals & 0.664 & 0.595 & 0.620 & 1 & & \\
\hline Semantic: Fruits & 0.514 & 0.507 & 0.551 & 0.651 & 1 & \\
\hline Semantic: Kitchen & 0.542 & 0.528 & 0.601 & 0.642 & 0.705 & 1 \\
\hline
\end{tabular}

We used linear regression analysis with a stepwise method to find the possible predictors of letter fluency. Education (illiterate, medium level, university), mother tongue (Farsi, others), and age could significantly predict the number of generated words. In semantic fluency, gender was also a significant predictor, in addition to education, mother tongue, and age (Table 5).

\section{Suggested cut-off points}

Table 6 suggests the possible cut-off points of the verbal fluency test for different genders, educational levels, and mother tongues. Therefore, we calculated the respective percentiles and presented them in Table 6. However, letter fluency in an illiterate population of the study was too low (0 or 1); therefore, it cannot be a useful clinical

Table 4. Number of generated words in different demographic subgroups

\begin{tabular}{|c|c|c|c|c|c|}
\hline Variables & Domain & Subgroups & Mean $\pm S D$ & Test Statistic & $\mathbf{P}$ \\
\hline \multirow{2}{*}{ Gender } & Letter & $\begin{array}{c}\text { Males } \\
\text { Females }\end{array}$ & $\begin{array}{l}8.0 \pm 3.8 \\
8.7 \pm 4.3\end{array}$ & $\mathrm{t}=1.90$ & 0.057 \\
\hline & Semantic & $\begin{array}{c}\text { Males } \\
\text { Females }\end{array}$ & $\begin{array}{l}17.3 \pm 5.5 \\
18.6 \pm 5.5\end{array}$ & $t=2.79$ & 0.005 \\
\hline \multirow{2}{*}{ Mother tongue } & Letter & $\begin{array}{l}\text { Farsi } \\
\text { Others }\end{array}$ & $\begin{array}{l}9.0 \pm 4.0 \\
6.9 \pm 3.7\end{array}$ & $t=5.55$ & $<0.001$ \\
\hline & Semantic & $\begin{array}{c}\text { Farsi } \\
\text { Others }\end{array}$ & $\begin{array}{l}19.4 \pm 5.0 \\
14.8 \pm 5.3\end{array}$ & $t=9.41$ & $<0.001$ \\
\hline \multirow{4}{*}{ Education } & Letter & $\begin{array}{l}\text { Illiterate } \\
\text { Preliminary } \\
\text { High School } \\
\text { University }\end{array}$ & $\begin{array}{l}4.4 \pm 2.7 \\
8.5 \pm 3.3 \\
8.8 \pm 3.2 \\
11.6 \pm 3.3\end{array}$ & $\mathrm{~F}=117.23$ & $<0.001^{*}$ \\
\hline & & Illiterate & $13.3 \pm 4.8$ & \multirow{3}{*}{$F=64.48$} & \multirow{3}{*}{$<0.001^{*}$} \\
\hline & Semantic & $\begin{array}{l}\text { Preliminary } \\
\text { High School }\end{array}$ & $\begin{array}{l}19.3 \pm 5.1 \\
18.2 \pm 4.4\end{array}$ & & \\
\hline & & University & $21.1 \pm 4.4$ & & \\
\hline
\end{tabular}

"In both domains: Illiterate<(Preliminary \& High School)<University. 
Table 5. Predicting number of words generated in letter and semantic fluency domains

\begin{tabular}{|c|c|c|c|c|c|c|}
\hline \multirow{2}{*}{ Variables } & \multirow{2}{*}{ Predictor } & \multicolumn{2}{|c|}{ Unstandardized Coefficients } & \multirow{2}{*}{$\begin{array}{l}\text { Standardized } \\
\text { Coefficients }\end{array}$} & \multirow{2}{*}{$t$} & \multirow{2}{*}{$\mathbf{P}$} \\
\hline & & B & Standard Error & & & \\
\hline \multirow{5}{*}{ Letter fluency } & Education & 3.256 & 0.757 & 0.574 & 15.5 & $<0.001$ \\
\hline & Mother tongue & 1.118 & 0.211 & 0.128 & 3.7 & $<0.001$ \\
\hline & Age & -0.034 & 0.302 & -0.108 & -3.0 & 0.003 \\
\hline & Constant & 2.456 & 0.012 & & 3.2 & 0.001 \\
\hline & \multicolumn{6}{|c|}{ Adjusted $R^{2}=0.430 ; F=126.3 ; P<0.001$} \\
\hline \multirow{6}{*}{$\begin{array}{l}\text { Semantic } \\
\text { fluency }\end{array}$} & Education & 3.174 & 0.305 & 0.411 & 10.4 & $<0.001$ \\
\hline & Mother tongue & 3.551 & 0.438 & 0.298 & 8.1 & $<0.001$ \\
\hline & Age & -0.043 & 0.017 & -0.099 & -2.5 & 0.011 \\
\hline & Gender & 1.013 & 0.398 & 0.092 & 2.5 & 0.011 \\
\hline & Constant & 10.414 & 1.111 & & & $<0.001$ \\
\hline & \multicolumn{6}{|c|}{ Adjusted $R^{2}=0.356 ; F=70.0 ; P<0.001$} \\
\hline
\end{tabular}

NEUR SCIENCE

Education: Illiterate=0, Medium level=1, University=2; Mother tongue: Farsi=1, Others=0; Gender: Female=1, Male=0

marker of deficit at this educational level. For those with primary or high school training, a cut-off point of 4 can be considered for a Persian-speaking male. One point can be decreased for non-Persian speaking males (score of 3), and one point increased for female subjects (score of 5) regardless of their mother tongue. Finally, for universitylevel education, a cut-off point of 7 can be considered for both ages regardless of their mother tongue.

For category fluency, 8 can be considered the cut-off point for illiterate male subjects. Three points should be decreased for people with a mother tongue other than Persian (score of 5 for both males and females), and 3 points should be increased for females with a Persian mother tongue (score of 11). For primary school and high school levels of education, a score of 12 can be equally fit for male and female subjects with a Persian mother tongue and female subjects with a mother tongue other than Persian. However, for males with another mother tongue, 3 scores should be decreased to find an appropriate cut-off point (score of 9).

The cut-off point of 12 can also be considered an appropriate point for university-level male subjects regardless of their mother tongue. For female subjects of this level of education, we can add three points to find a suggested cut-off point of 15 for both Persian and other mother tongues.

\section{Discussion}

We conducted this study to prepare a Persian version of the verbal fluency test in Iran. We selected three letters $(/ \mathrm{p} /, / \mathrm{d} /, / \mathrm{J} /)$ and three categories (animals, fruits, and kitchen appliances) and calculated the mean number of generated words in each set, considering the influential variables. The Mean \pm SD numbers of generated words in letter fluency and semantic fluency were $8.3 \pm 4.1$ and $18.0 \pm 5.5$, respectively. We found that verbal fluency, in both letter and semantic tests, is related to several factors. In letter fluency, age, educational level, and mother tongue, and regarding semantic fluency, age, gender, education level, and mother tongue were effective factors in the number of generated words.

The number of generated words in the letter fluency task in our study is considerably lower than the studies on English speaking populations using the F-A-S letter fluency task (12 in our study vs 15.2 in F-A-S studies) but comparable to the findings of a study by Olabarrieta-Landa et al. using F-A-S in 3977 healthy individuals from Latin America (7.3 to 13.9) (Olabarrieta-Landa et al., 2015). It should be noted that, in comparing the results of verbal fluency between two different languages, one should consider the number of words that begin in each language with the given letter. Verbal fluency performance is associated with the frequency of words beginning with a given 
Table 6 . The $5^{\text {th }}$ and $10^{\text {th }}$ percentiles of generated words in each category

\begin{tabular}{|c|c|c|c|c|c|c|}
\hline \multicolumn{3}{|c|}{ Subgroups } & \multicolumn{2}{|c|}{ Letter } & \multicolumn{2}{|c|}{ Category } \\
\hline Gender & Language & Education & $5^{\text {th }}$ Percentile & $10^{\text {th }}$ Percentile & $5^{\text {th }}$ Percentile & $10^{\text {th }}$ Percentile \\
\hline \multirow{6}{*}{ Male } & & Illiterate & 0 & 2 & 7 & 8 \\
\hline & Farsi & Medium & 3 & 4 & 13 & 14 \\
\hline & & University & 7 & 7 & 12 & 15 \\
\hline & & Illiterate & 0 & 1 & 4 & 5 \\
\hline & Other & Medium & 2 & 3 & 9 & 10 \\
\hline & & University & 6 & 7 & 14 & 14 \\
\hline \multirow{6}{*}{ Female } & & Illiterate & 0 & 1 & 11 & 11 \\
\hline & Farsi & Medium & 4 & 5 & 12 & 14 \\
\hline & & University & 6 & 7 & 15 & 17 \\
\hline & & Illiterate & 1 & 2 & 4 & 5 \\
\hline & Other & Medium & 4 & 5 & 12 & 12 \\
\hline & & University & 6 & 7 & 17 & 17 \\
\hline
\end{tabular}

letter in a language (Kempler, D., Teng,Dick,Taussig, \& Davis, 1998). Therefore, the observed difference could be partly due to the difference in letter frequency and the number of words that begin with the specified letters in each language. For example, $\mathrm{A}$ and $\mathrm{S}$ are among the letters with the highest frequency in English, and $\mathrm{F}$ has a medium frequency. However, P, D, and Sh in Persian are among the medium frequency letters.

In another recently published study on Persian participants (Hasemian-Shirvan et al., 2018), 3 letters (Pe standing for $/ \mathrm{p} /$, Meem for $/ \mathrm{m} /$, and Kaaf for $/ \mathrm{k} /$ ) suggested as the highest frequency in word production (12 on average) and had been chosen for Persian phonemic VFT. Participants were assessed with the 3 selected letters $(/ \mathrm{p} /$ : $12.28 \pm 3.607, / \mathrm{m} /:$ : $12.54 \pm 3.907$, and $/ \mathrm{k} /: 12.48 \pm 3.708)$ and 3 semantic categories (animal: $21.67 \pm 5.119$, supermarket: $21.19 \pm 4.907$, and fruit: $19.58 \pm 4.439$ ) with 1-minute time limitation for each test. It was a preliminary report and performed only on 100 subjects. In contrast to this study, we did not use letters that can be used at the beginning of present continuous verb forms (Meem for $/ \mathrm{m} /$ ) or common inappropriate words (Kaaf for $/ \mathrm{k} /$ ). The mean word production is lower in our study, and this may be partly due to lower educational levels and including illiterates in the current study.
In the study by Ghoreishi and Azimian (2014), in 120 normal Persian speakers using $/ \varphi / \mathrm{p} /$ and $/ \mathrm{\rho} / / \mathrm{m} /$ in 30 seconds, the Mean \pm SD number of words was $9.16 \pm 3.04$, which seems higher than the current study. However, $85 \%$ of the study participants had education above 12 years, which is much higher than our study and might have resulted in higher scores in Ghoresishi and Azimian's study. In another study, Mohammadisabet et al. performed an F-A-S letter fluency task on 90 normal elderlies, and the mean number of generated words was 18 for males and 19.42 for females (Mohammadisabet, Yadegari, \& Foroughan, 2016). Although not directly mentioned, the authors have summed up the number of generated words with the three letters and not divided them by three. This might be the reason for the high score reported in this research. Malek, Hekmati, Amiri, Pirzadeh, \& Gholizadeh (2013), in a study among bilingual (Persian-Turkish) adolescents (11-18 years old) using $/ \mathrm{m} /, / \mathrm{d} /$, and $/ \mathrm{b} /$ for letter fluency, reported mean scores of $9.7,9.3$, and 9.2 , respectively.

In semantic fluency, the mean number of generated words in our study was 16.5. In other studies, it ranges from 16.97 to 19.3 for the animal semantic test in Mexico, Spain, and Argentina (Benito-Cuadrado, Esteba-Castillo, Böhm, Cejudo-Bolivar, \& Peña-Casanova, 2002; Alamo, et al., 1999; Butman, Allegri, Harris, \& Drake, 2000). We should keep in mind that the number 
of syllables of the names of animals and objects varies between different languages and the semantic fluency score is better in languages with shorter animal names (predominantly 1 syllable) than the languages where animal names are longer ( 2 and 3 syllables per word) (Kemper, 1998). In the Persian language, the animal names are generally short (1 syllable).

Ghoreishi and Azimian calculated animal and fruit naming in 30 seconds, and the Mean \pm SD score for semantic fluency was $13.66 \pm 2.97$ (Ghoreishi, 2014). Mohammadisabet et al. (2016) reported semantic fluency in the elderly with mean values of 27.55 and 30.24 for males and females, respectively. As previously mentioned, participants of the former study had a higher level of education than the current study, and the score in the latter study is calculated by adding up the number of fruits and animals generated in one minute. Additionally, Malek et al., in a study on bilingual Persian adolescents, showed that the score of semantic fluency for the two categories of animal and supermarket stuff were 14.76 and 13.57, respectively (Malek, et al., 2013).

We also found that verbal fluency, for both letter and semantic tasks, is related to several factors. Age, educational level, and mother tongue are associated with letter fluency. Also, age, gender, education level, and mother tongue are associated with semantic fluency. The findings on the effect of educational level (Ostrosky-Solis et al., 2007; Kim et al., 2013; Zarino, Crespi, Launi, \& Casarotti, 2014; Chávez-Oliveros, 2015; Obeso, Casabona, Bringas, Álvarez, \& Jahanshahi, 2012) and age (Kim et al., 2013; Zarino et al., 2014; Obeso et al., 2012) on verbal fluency is consistent with previous studies.

However, the results of the studies are inconsistent regarding the effect of gender on verbal fluency. Some studies have suggested that gender is a contributing factor (Zarino et al., 2014; Chávez-Oliveros, Rodríguez-Agudelo, Acosta-Castillo, García-Ramírez, de la Torre, \& Sosa-Ortiz, 2015), while others have not replicated this finding (Kim et al., 2013; Zarino et al., 2014; Chávez-Oliveros, 2015; Obeso, I., Casabona, Bringas, Álvarez, \& Jahanshahi, 2012). In our study, gender was only related to semantic fluency and not letter fluency.

We found that bilingualism is also an essential factor. Previous studies also found bilingualism as an effective factor, especially in semantic fluency task (Gollan, Montoya, \& Werner, 2002; Rosselli et al., 2000; Loewenstein, Argüelles, Argüelles, \& Linn-Fuentes, 1994). The other issue that should be considered is differentiating early and late bilingualism. Some studies found better verbal fluency results in late bilingualism than in early one (Rosselli et al., 2000; Portocarrero, Burright, \& Donovick, 2007). All of our subjects were early bilinguals.

\section{Conclusion}

Verbal fluency tests are useful measures of cognitive function and study acquired language impairments. For clinical usage of the verbal fluency test, we suggest clinicians consider the following points in assessing subjects to have more accurate and reliable results.

Assess fluency of each subject for all of the three letters (not a single letter) and all of the three categories (not a single category) and calculate an average for letter fluency and another average for category fluency.

The current study has several limitations that should be considered. Our sample included only a limited number of subjects with mother tongues other than Persian. Therefore, caution should be made when using the findings for the subjects of these subgroups, especially those with the non-Azari mother tongue. The task needs to be normalized in these populations in future studies. Furthermore, we did not include subjects under 18 or over 65 years old. These age groups should be evaluated in separate studies that include only children and adolescents in specific age groups or elderly individuals.

\section{For illiterate subjects}

Letter fluency: as it is developed in this study, it does not seem to be valid and should not be used.

Category fluency: cut-off point of 8 can be considered for male and 11 for female subjects. For those with a non-Persian mother tongue, a cut-off point of 5 can be considered for both men and women.

\section{For those with preliminary or high school training}

Letter fluency: cut-off point of 4 can be considered for men with a Persian mother tongue, 3 for men with a nonPersian mother tongue, 5 for female subjects regardless of their mother tongue.

Category fluency: cut-off point of 12 for men and women with Persian mother tongue, and for those with non-Persian mother tongue, a score of 9 and 12 can be used for men and women, respectively.

\section{For university-level education}

Letter fluency: a cut-off point of 7 can be considered for both ages regardless of their mother tongue.

Category fluency: cut-off point of 12 can be considered for male subjects regardless of their mother tongue. For female subjects, a cut-off point of 15 can be suggested regardless of their mother tongue. 


\section{Ethical Considerations}

\section{Compliance with ethical guidelines}

The study was approved by the Ethics Committee of Iran University of Medical Sciences. The study's purpose was explained to all participants, and they gave consent for participating in the study and having their voices recorded.

\section{Funding}

This research did not receive any grant from funding agencies in the public, commercial, or non-profit sectors.

\section{Authors' contributions}

All authors equally contributed to preparing this article.

\section{Conflict of interest}

The authors declared no conflict of interest

\section{Acknowledgments}

We would like to thank all subjects that helped us in the study.

\section{References}

Álamo, C. D., Mir, M. L., Olivares, T., Barroso, J., \& Nieto, A. (1999) Efecto de la edad,nivel educativo y estado cognitivo general sobre la fluidez verbal enhispanoparlantes. Datos normativos preliminares. First International Congress on Neuropsychology [Internet]. 1999. http://www.uninet.edu/union99/congress/ libs/val/v01.html. Fecha última consulta:28.03.05.54.

Benito-Cuadrado, M. M., Esteba-Castillo, S., Böhm, P., CejudoBolivar, J., \& Peña-Casanova, J. (2002). Semantic verbal fluency of animals: A normative and predictive study in a Spanish population. Journal of Clinical and Experimental Neuropsychology, 24(8), 1117-22. [DOI:10.1076/jcen.24.8.1117.8376] [PMID]

Butman, J., Allegri, R. F., Harris, P., \& Drake, M. (2000). Fluencia verbal en español. Datos normativos en Argentina [Spanish verbal fluency. Normative data in Argentina]. Medicina (B Aires), 60(5 Pt 1):561-4. [PMID]

Chávez-Oliveros, M., Rodríguez-Agudelo, Y., Acosta-Castillo, I., García-Ramírez, N., de la Torre, G. R., \& Sosa-Ortiz, A. L. (2015). Semantic verbal fluency in elderly Mexican adults: Reference values. Neurología (English Edition), 30(4), 189-94. [DOI:10.1016/j.nrleng.2015.03.001] [PMID]

Ghoreishi, Z. S., Azimian, M., Khorrami Banaraki, B., Alaghband Rad, J., Rafiee, S. M., Salavati, M., et al. (2014). Lexical access in Persian normal speakers: Picture naming, Verbal fluency and Spontaneous speech. Iranian Rehabilitation Journal, 12(2), 16-20. http://iri.uswr.ac.ir/article-1-367-en.html
Gollan, T. H., Montoya, R. I., \& Werner, G. A. (2002). Semantic and letter fluency in Spanish-English bilinguals. Neuropsychology, 16(4), 562-76. [DOI:10.1037/0894-4105.16.4.562] [PMID]

Hasemian-Shirvan, E., Shirazi, S. M., Aminikhoo, M., Zareaan M., \& Ekhtiari, H. (2018). Preliminary normative data of Persian phonemic and semantic verbal fluency test. Iranian Journal of Psychiatry, 13(4), 288-95. [PMID] [PMCID]

Kempler, D., Teng, E. L., Dick, M., Taussig, I. M., \& Davis, D. S. (1998). The effects of age, education, and ethnicity on verbal fluency. Journal of the International Neuropsychological Society, 4(6), 531-8. [DOI:10.1017/S1355617798466013] [PMID]

Kim, B. J., Lee, C. S., Oh, B. H., Hong, C. H., Lee, K. S., Son, S. J., et al. (2013). A normative study of lexical verbal fluency in an educationally-diverse elderly population. Psychiatry Investigation 10(4), 346-51. [DOI:10.4306/pi.2013.10.4.346] [PMID] [PMCID]

Loewenstein, D. A., Argüelles, T., Argüelles, S., \& LinnFuentes, P. (1994). Potential cultural bias in the neuropsychological assessment of the older adult. Journal of Clinical and Experimental Neuropsychology, 16(4), 623-9. [DOI:10.1080/01688639408402673] [PMID]

Malek, A., Hekmati, I., Amiri, S., Pirzadeh, J., \& Gholizadeh, H. (2013). Designing and standardization of Persian version of verbal fluency test among Iranian bilingual (Turkish-Persian) adolescents. Journal of Analytical Research in Clinical Medicine, 1(1), 32-42. [DOI:10.1155/2013/165193] [PMID] [PMCID]

Mohammadi-Sabet, V., Yadegari, F., \& Foroughan, M. (2016). [Verbal fluency performance in normal elderly Persian speakers (Persian)]. Salmand: Iranian Journal of Ageing, 11(2), 290-9. [DOI:10.21859/sija-1102290]

Obeso, I., Casabona, E., Bringas, M. L., Álvarez, L., \& Jahanshahi, M. (2012). Semantic and phonemic verbal fluency in Parkinson's disease: Influence of clinical and demographic variables. Behavioural Neurology, 25(2), 111-8. [DOI:10.1155/2012/673610] [PMID] [PMCID]

Olabarrieta-Landa, L., Rivera, D., Galarza-del-Angel, J., Garza, M. T., Saracho, C. P., Rodriguez, W., et al. (2015). Verbal fluency tests: Normative data for the Latin American Spanish speaking adult population. NeuroRehabilitation, 37(4), 515-61. [DOI:10.3233/NRE-151279] [PMID]

Ostrosky-Solis, F., Gutierrez, A. L., Flores, M. R., \& Ardila, A. (2007). Same or different? Semantic verbal fluency across Spanishspeakers from different countries. Archives of Clinical Neuropsychology, 22(3), 367-7. [DOI:10.1016/j.acn.2007.01.011] [PMID]

Patterson, J. (2011). Verbal fluency. In J. S. Kreutzer, J. DeLuca, \& B. Caplan (Eds.), Encyclopedia of Clinical Neuropsychology. New York: Springer. (pp. 2603-6). [DOI:10.1007/978-0-387-79948-3_1423]

Portocarrero, J. S., Burright, R. G., \& Donovick, P. J. (2007). Vocabulary and verbal fluency of bilingual and monolingual college students. Archives of Clinical Neuropsychology, 22(3), 415-22. [DOI:10.1016/j.acn.2007.01.015] [PMID]

Rosselli, M., Ardila, A., Araujo, K., Weekes, V. A., Caracciolo, V. Padilla, M., et al. (2000). Verbal fluency and repetition skills in healthy older Spanish-English bilinguals. Applied Neuropsychology, 7(1), 17-24. [DOI:10.1207/S15324826AN0701_3] [PMID]

Zarino, B., Crespi, M., Launi, M., \& Casarotti, A. (2014). A new standardization of semantic verbal fluency test. Neurological Sciences, 35(9), 1405-11. [DOI:10.1007/s10072-014-1729-1] [PMID] 
This Page Intentionally Left Blank 